\title{
ANÁLISE DA DEMANDA FONOAUDIOLÓGICA DE UM HOSPITAL DE REFERENNCIA EM CARDIOPNEUMOLOGIA
}

ANALYSIS OF THE SPEECH THERAPY NEEDS IN A REFERRAL HOSPITAL IN CARDIOPNEUMOLOGY

ANÁLISIS DE LA DEMANDA FONOAUDIOLÓGICA EN UN HOSPITAL DE REFERENCIA EN CARDIONEUMOLOGÍA

Bruna Rafaelle Pereira Ibiapina Coelho ${ }^{1}$ Luana Pereira Ibiapina Coelho 2

Elizabeth Zayra Torres Sousa ${ }^{3}$

Ana Virgínia Sales Monte Costa 4

Antonio Tiago da Silva Souza ${ }^{5}$

Camila Fernandes Mendes 6

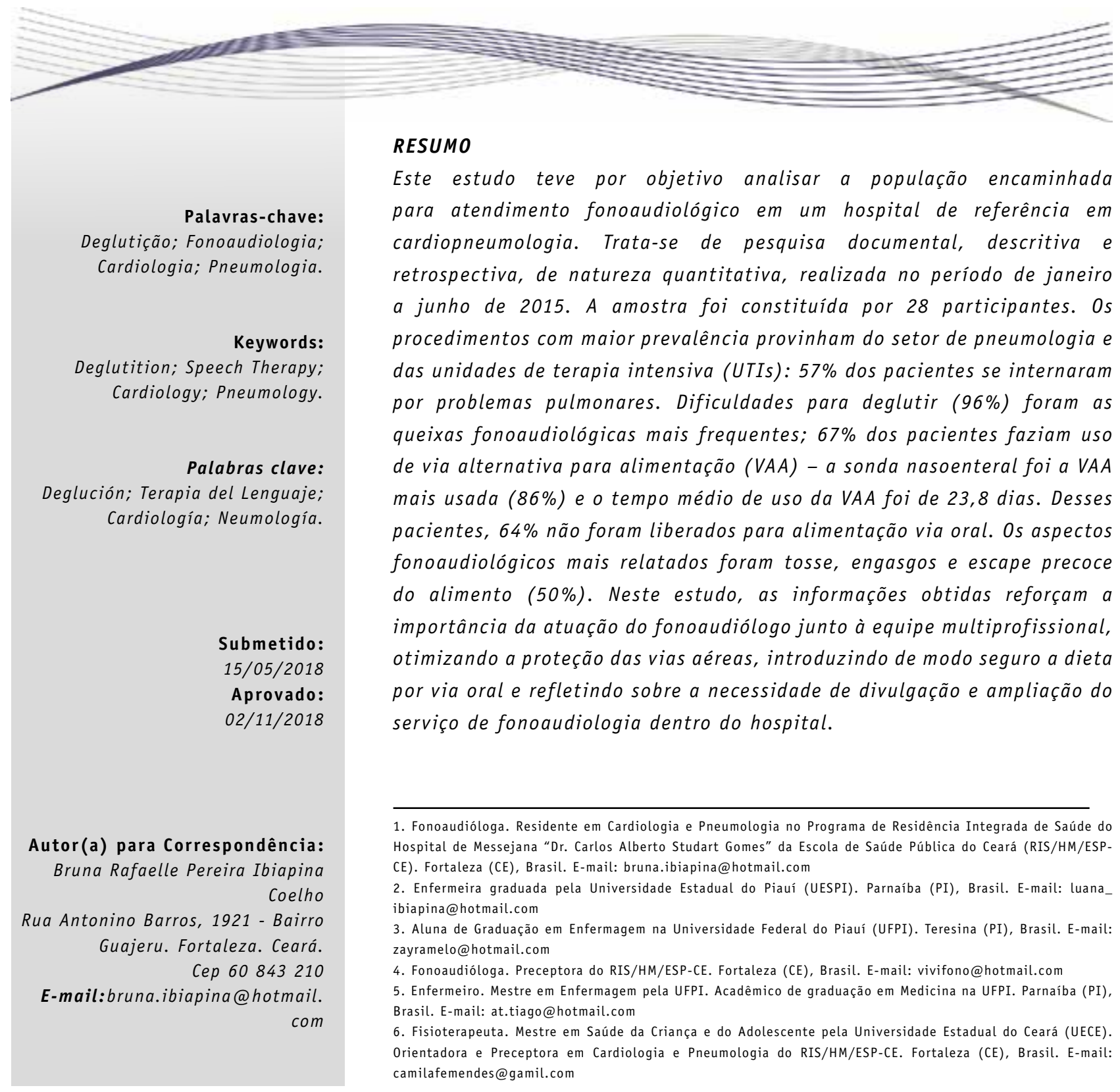

12 - SANARE, Sobral - V.18 n.01,p.12-21, Jan./Jun. - 2019 


\section{ABSTRACT}

This study aimed to analyze the population seeking speech therapy in a referral hospital in cardiopneumology. This is a documentary, descriptive, and retrospective research, of a quantitative nature, carried out within the period from January to June 2015. The sample consisted of 28 participants. The most prevalent procedures took place in the pulmonology sector and the intensive care units (ICUs): 57\% of the patients were hospitalized for lung issues. Difficulties in swallowing (96\%) were the most frequent speech therapy complaints; $67 \%$ of the patients used an alternative route for feeding (ARF) - the nasoenteral tube was the most used ARF ( $86 \%$ ) and the mean time of ARF use was 23.8 days. Out of these patients, $64 \%$ were not allowed to have oral feeding. The most commonly reported speech therapy issues were coughing, choking, and early food escape (50\%). In this study, the information obtained reinforces the importance of the speech therapist's work along with the multiprofessional team, increasing airway protection, safely introducing the oral diet, and thinking through the need to disseminate and expand the speech therapy services within the hospital.

\section{RESUMEN}

Este estudio tuvo como objetivo analizar la población que buscaba terapia del lenguaje en un hospital de referencia en cardioneumología. Se trata de una investigación documental, descriptiva y retrospectiva, de carácter cuantitativo, realizada en el período comprendido entre enero y junio de 2015. La muestra estuvo compuesta por 28 participantes. Los procedimientos más prevalentes se realizaron en el sector de neumología y en las unidades de cuidados intensivos (UCIS): el 57\% de los pacientes fueron hospitalizados por problemas pulmonares. Las dificultades para tragar (96\%) fueron las quejas más frecuentes en la terapia del lenguaje; el 67\% de los pacientes utilizaron una ruta alternativa para la alimentación (RAA) - el tubo nasoenteral fue la RAA más utilizada (86\%) y el tiempo medio de uso de la RAA fue de 23,8 días. De estos pacientes, al 64\% no se les permitió recibir alimentación oral. Los aspectos de terapia del lenguaje más comúnmente informados fueron la tos, la estrangulación y el escape prematuro del alimento (50\%). En este estudio, la información obtenida refuerza la importancia del trabajo del terapeuta del lenguaje junto con el equipo multiprofesional, aumentando la protección de las vías respiratorias, introduciendo de manera segura la dieta oral y pensando en la necesidad de difundir y ampliar los servicios de terapia del lenguaje dentro del hospital.

\section{INTRODUÇÃO}

As doenças cardiovasculares contribuem significativamente como grupo causal de mortalidade em todas as regiões do Brasil. Em 2000, essas doenças foram responsáveis pela principal alocação de recursos públicos em hospitalizações no país e foram a terceira causa de permanência hospitalar prolongada. Entre 1991 e 2000 , os custos hospitalares atribuídos a elas aumentaram $176 \%{ }^{1}$.

As doenças cardiovasculares apresentam alta prevalência e complicações associadas, como o acidente cerebrovascular encefálico (ACE), a insuficiência cardíaca (IC) e o infarto agudo do miocárdio. Tais doenças têm considerável impacto na morbimortalidade das populações e são as principais causas de óbito no Brasil há algumas décadas².

$\mathrm{Na}$ vigência do pós-operatório das cirurgias cardíacas de alguns pacientes, estudos vêm alertando sobre a presença de distúrbios da deglutição, devido ao fato de eles se submeterem a procedimentos como intubação orotraqueal (I0T), ventilação mecânica (VM), circulação extracorpórea (CEC), traqueostomia (TQT) e ecocardiograma transesofágico transoperatório (ETT), que podem ocasionar alterações na dinâmica da deglutição ${ }^{3}$.

Segundo a Organização Mundial da Saúde (OMS), entende-se por doenças respiratórias crônicas as enfermidades crônicas de vias aéreas e de outras estruturas dos pulmões. Dentre as mais comuns se encontram a asma, a doença pulmonar obstrutiva crônica (DPOC), as doenças respiratórias alérgicas, as moléstias pulmonares ocupacionais e a hipertensão pulmonar.

A DPOC vem tendo grande destaque no meio médico nos últimos anos, tendo em vista a tomada de consciência de sua importância como fator de morbimortalidade. No Brasil, ela já ocupa a $5^{\text {a }}$ posição entre as causas de óbito e envolve a internação de 290 mil pacientes anualmente, trazendo enorme gasto ao sistema de saúde do país ${ }^{5,6}$.

As complicações pulmonares pós-operatórios (CPPO) ocorrem em $25 \%$ a $50 \%$ dos procedimentos cirúrgicos de grande porte. A ocorrência de CPPO está 
ligada à existência dos fatores de risco descritos na literatura, tais como: a) idade avançada (> 60 anos); b) presença de doença pulmonar prévia, como DPOC ou outras comorbidades; c) classificação da American Society of Anesthesiologists (ASA) maior ou igual a II; d) tabagismo ativo; e) obesidade; f) desnutrição; g) tipo de anestesia; h) tempo de cirurgia ( $>3$ horas); i) tipo de cirurgia; j) valores espirométricos anormais; k) capacidade diminuída ao exercício; e l) tempo de internação pré-operatória prolongado ${ }^{7}$.

Pacientes com DPOC tendem a interromper sua respiração durante a deglutição, retomando-a na fase inspiratória e podendo, assim, aumentar o risco para aspiração. Em um estudo realizado com 14 pacientes com DPOC, relatou-se que os participantes apresentaram redução da habilidade de limpeza laríngea e de proteção da via aérea, resultando em aumento do risco para aspiração. Em outro estudo, relatou-se que pacientes com DPOC apresentavam alterações no reflexo de deglutição, sendo tal alteração sugestiva de fator de risco para a exacerbação da doença ${ }^{8}$.

Pacientes com doenças crônicas pulmonares podem ser suscetíveis a apresentar alteração na coordenação entre deglutição e respiração, devido a alterações funcionais ventilatórias ${ }^{9}$.

A fonoaudiologia hospitalar atua com o paciente ainda no leito, em caráter precoce, preventivo, intensivo, pré e pós-cirúrgico, proporcionando respaldo técnico e prático à equipe interdisciplinar em que atua ao esclarecer que seu maior objetivo é impedir ou diminuir as sequelas que a patologiabase possa deixar ${ }^{10}$.

A efetividade da intervenção fonoaudiológica tem sido demonstrada por meio de estudos, com associação entre menor tempo de internação hospitalar e realização do trabalho fonoaudiológico. Mesmo com pesquisas que mostram os benefícios dessa intervenção, muitos serviços não dispõem dessa prática, tampouco realizam encaminhamento dessa população de risco para acompanhamento fonoaudiológico precoce ${ }^{11,12}$.

0 objetivo da reabilitação no atendimento a pacientes com distúrbios da deglutição consiste na estabilização do estado nutricional e na eliminação dos riscos de complicações clínicas decorrentes da aspiração laringotraqueal ${ }^{13}$.

A deglutição tem como função fundamental a propulsão do alimento da boca para o estômago. É um processo contínuo no qual todos os músculos

\section{A disfagia (...) causa alto indice de morbimortalidade.}

relacionados com a cavidade oral entram em açã $0^{11}$. A deglutição se divide em três fases: a) oral; b) faríngea; e c) esofágica ${ }^{14,15}$.

A disfagia é um prejuízo no funcionamento de qualquer fase da deglutição. A coordenação entre deglutição e respiração se mostra essencial para prevenir a aspiração pulmonar e para manter a nutrição e a hidratação adequadas ${ }^{7}$.

Do ponto de vista etiológico, as disfagias podem ser divididas em: a) neurogênicas, quando causadas por alguma afecção no sistema nervoso central e/ou periférico; e b) mecânicas, quando desencadeadas por alterações anatômicas de qualquer origem. Quanto à gravidade, podem ser classificadas em leves, moderadas e severas ${ }^{14}$.

Pacientes com disfagia aumentam os custos de internação, prolongam seu tempo de internação e expõem-se ao risco de desnutrição, desidratação e complicações pulmonares, devido à broncoaspiração. A disfagia é considerada por vários autores um problema de saúde pública, uma vez que afeta grande parte da população e causa alto índice de morbimortalidade ${ }^{15}$.

A aspiração pulmonar pode ser fatal e a má nutrição pode ocorrer quando a alteração não é diagnosticada de modo correto, reduzindo a imunidade do paciente e deixando-o mais suscetível a contrair doenças ${ }^{13}$.

À medida que seu campo de atuação se amplia, novas especialidades surgem na área - uma das mais recentes é a disfagia, regulamentada pela Resolução do Conselho Federal de Fonoaudiologia (CFFa) $n$. 356, de 6 de dezembro de 2008, que “dispõe sobre a competência técnica e legal do fonoaudiólogo para atuar nas disfagias orofaríngeas". Denomina-se disfagia o distúrbio de deglutição que acomete todo o trato digestivo, que vai da boca até o estômago ou parte deste ${ }^{14}$.

Caracterizar o público que evolui com necessidade de acompanhamento fonoaudiológico se mostra importante para evidenciar os indivíduos que têm avançado comprometimento da deglutição.

Assim, esta pesquisa teve como finalidade 
analisar a população encaminhada para atendimento fonoaudiológico em um hospital de referência em cardiopneumologia. Secundariamente, objetivou-se relatar a procedência dos encaminhamentos, especificar a queixa fonoaudiológica e descrever os aspectos fonoaudiológicos identificados no período da solicitação do parecer fonoaudiológico.

\section{MÉTODOS}

Estudo documental, descritivo e retrospectivo, de natureza quantitativa, realizado por meio da análise de prontuários de pacientes com solicitação de parecer fonoaudiológico no período de janeiro a junho de 2015.

Foram coletados dados referentes a sexo, idade, procedimentos dos encaminhamentos (por unidade, setor e profissional solicitante), motivo da internação, se realizou procedimento cirúrgico, tempo de espera por atendimento fonoaudiológico, queixa fonoaudiológica, tempo e via alternativa para alimentação (VAA) utilizada, se for liberada alimentação via oral, aspectos fonoaudiológicos, uso de oxigenoterapia e tipo de via aérea artificial.

As informações foram registradas em um instrumento para coleta de dados desenvolvido no âmbito desta pesquisa. Excluíram-se os pacientes cujos prontuários não foram localizados ou estavam incompletos quanto às variáveis adotadas pelo estudo. Assim, dos 59 indivíduos identificados inicialmente, 28 foram selecionados para compor a amostra.

Os dados foram armazenados no software estatístico Microsoft Excel 2013, versão 15.0, sendo posteriormente consolidados para apresentação por médias e frequências.

Esta pesquisa foi aprovada pelo Comitê de Ética em Pesquisa do Hospital de Messejana "Dr. Carlos Alberto Studart Gomes" da Escola de Saúde Pública do Ceará (HM/ESP-CE), sob o Parecer n. 1.418.093/2016.

\section{RESULTADOS}

Dos 28 participantes da pesquisa, $64 \%$ eram do gênero masculino. Quanto à faixa etária, predominaram pacientes idosos (65 anos em média). Desses sujeitos, $82 \%$ procediam de Fortaleza-CE.

A maior parte dos pareceres fonoaudiológicos era solicitada do setor de pneumologia, como se observa na Figura 1.

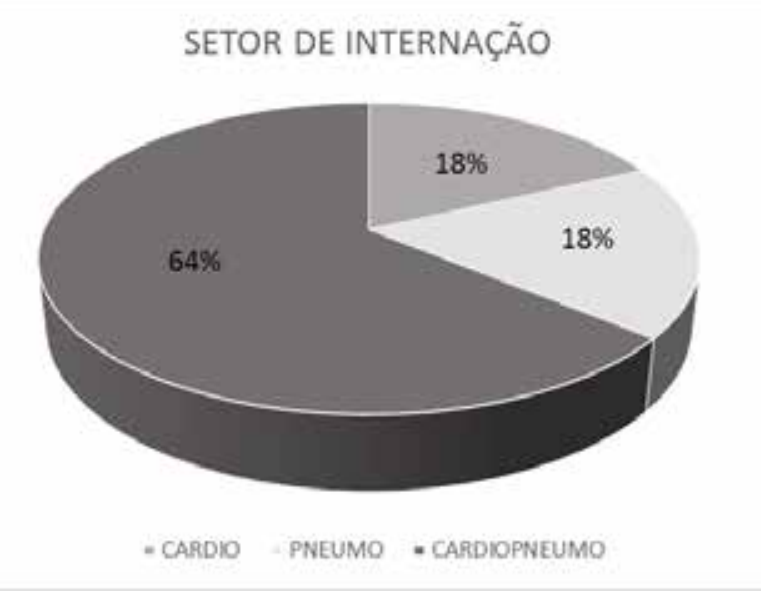

Figura 1. Solicitação de parecer fonoaudiológico por setor de internação $(n=28)$. Fortaleza, 2015.

Fonte: Elaborada pelos autores.

Em relação à distribuição dos pareceres fonoaudiológicos por unidades, $57 \%$ provieram das unidades de terapia intensiva (UTIs); $39 \%$ das enfermarias; e $4 \%$ do setor de emergência.

Quanto ao motivo de internação, verificou-se que $84 \%$ dos pacientes se internaram por problemas respiratórios e $16 \%$ por problemas cardíacos. Dos pacientes analisados, $57 \%$ realizaram algum tipo de procedimento cirúrgico.

0 tempo de espera para atendimento fonoaudiológico foi subdividido em dias: $71 \%$ dos pacientes foram avaliados em menos de 24 horas, $14 \%$ esperaram de 1 a 3 dias e $14 \%$ aguardaram mais de 3 dias. Dentre os profissionais que mais solicitaram parecer fonoaudiológico se destacam os médicos $(93 \%)$ e os nutricionistas (7\%).

As queixas fonoaudiológicas mais frequentes foram dificuldades para deglutir (96\%), relatadas pelos profissionais ao solicitarem parecer, como ilustra a Figura 2.

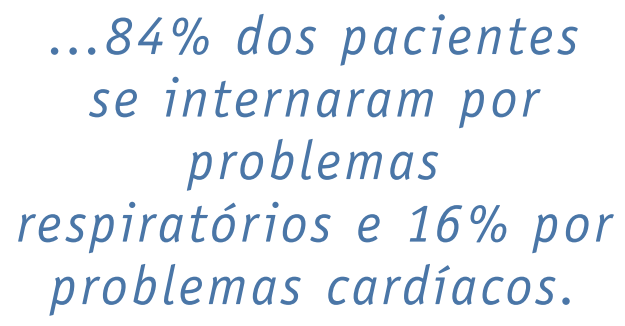




\section{QUEIXA FONOAUDIOLÓGICA}

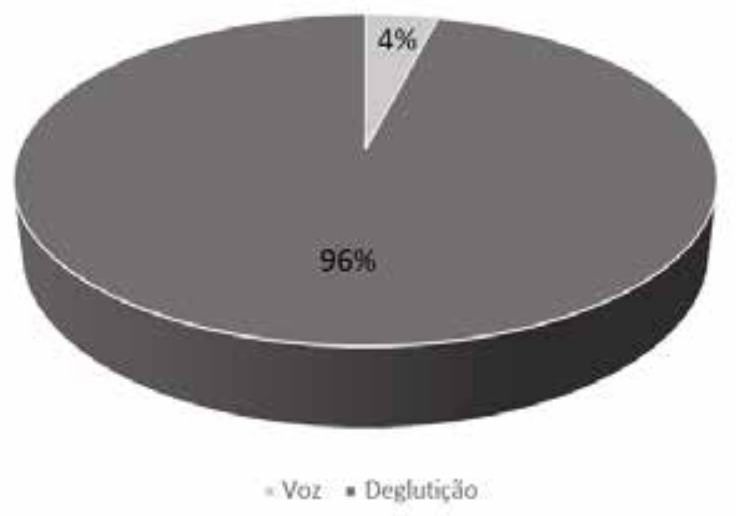

Figura 2. Principais queixas fonoaudiológicas no parecer fonoaudiológico $(n=28)$. Fortaleza, 2015. Fonte: Elaborada pelos autores.

No tocante às VAAs, $68 \%$ dos pacientes faziam uso desse recurso. A sonda nasoenteral (SNE) foi a VAA mais prevalente (57\%) - como mostra a Figura 3. 0 tempo médio de uso da VAA foi de 23,8 dias.

\section{Via Alternativa de Alimentação}

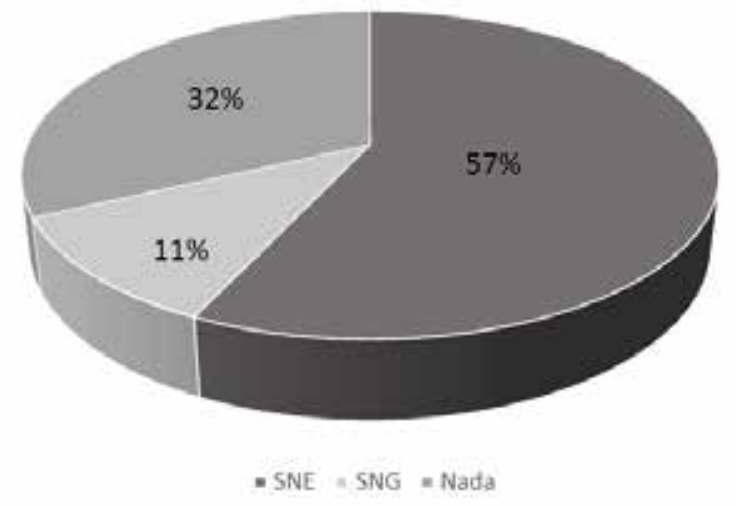

Figura 3. Distribuição da via alternativa utilizada no momento da avaliação $(n=28)$. Fortaleza, 2015. Fonte: Elaborada pelos autores.

Como mostra a Figura 4, 64\% dos pacientes analisados não foram liberados para alimentação via oral.

\section{Liberação de Alimentação por Via Oral}

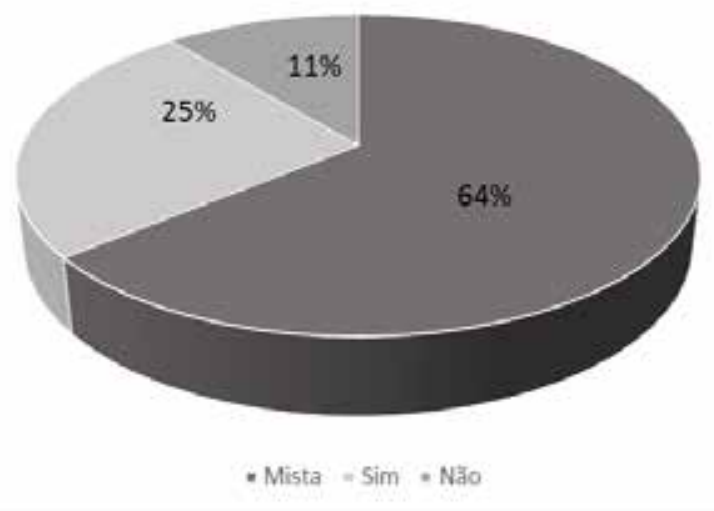

Figura 4. Distribuição quanto à liberação para alimentação via oral $(n=28)$. Fortaleza, 2015. Fonte: Elaborada pelos autores.

Dos pacientes avaliados, $61 \%$ faziam uso de oxigenoterapia, $36 \%$ deles por meio de cateter $0_{2}$, $18 \%$ em VM e $7 \%$ em ventilação não invasiva.

Quanto aos aspectos fonoaudiológicos mais descritos na avaliação, identificou-se tosse, engasgos e escape precoce do alimento, observados em $50 \%$ dos pacientes (Figura 5).

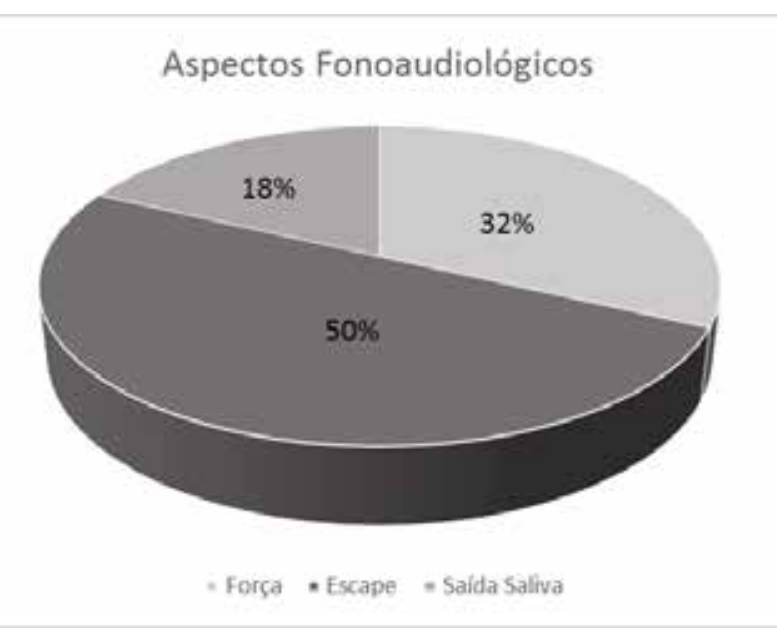

Figura 5. Distribuição quanto aos principais aspectos fonoaudiológicos na avaliação $(n=28)$. Fortaleza, 2015.

Fonte: Elaborada pelos autores.

\section{DISCUSSÃ 0}

Neste estudo, por meio do levantamento de prontuários, traçou-se uma análise da demanda fonoaudiológica em um hospital de referência em cardiopneumologia no período de janeiro a junho de 2015. 0 fato do número de atendimentos ter 
sido reduzido nesse intervalo pode justificar-se pelo desconhecimento dos profissionais acerca da existência do serviço de fonoaudiologia no hospital em estudo.

Aspectos como escolaridade, ocupação e estado civil não constam nos registros dos prontuários, mostrando-se impossivel uma análise criteriosa deles. Outro entrave durante a coleta de dados foi a dificuldade de localizar os prontuários dos pacientes selecionados.

No que diz respeito à análise da demanda, houve predomínio do sexo masculino. Segundo a Política Nacional de Atenção Integral à Saúde do Homem, observa-se que os sujeitos do sexo masculino são mais vulneráveis a doenças, sobretudo no que se refere a enfermidades graves e crônicas; nesse âmbito, a doença é considerada sinal de fragilidade, medo, dificuldade de acesso aos serviços sociais etc., por isso os homens não se preocupam tanto com sua própria saúde ${ }^{16}$.

Quanto à idade, houve maior incidência da população idosa (65 anos em média). Isso ocorre devido à sua maior suscetibilidade fisiológica e imunológica, particularmente às infecções, contribuindo para a redução de sua capacidade física e biológica ${ }^{17}$.

Esta pesquisa evidenciou prevalência de pacientes que moram na capital, porém, indivíduos que residem em outras cidades também são atendidos no hospital, possivelmente devido à carência do atendimento na rede pública de saúde de seus municípios ${ }^{18}$.

As demandas por serviços de saúde resultam da conjugação de fatores sociais, individuais e culturais prevalentes na população. A oferta está relacionada à disponibilidade, ao tipo e à quantidade de serviços e recursos destinados à atençã ${ }^{19}$.

0 motivo de internação com maior prevalência foi de paciente com problemas respiratórios, sendo o setor de pneumologia o que mais demandou atendimento fonoaudiológico. No entanto, notase que, para a população com 60 anos ou mais, esse indicador se apresenta mais elevado para internações por doenças respiratórias de pacientes do sexo masculino; acrescenta-se, ainda, que as hospitalizações por doenças respiratórias têm sido responsáveis, nos últimos anos, por $10 \%$ ou $11 \%$ do total de internações da população acima de 60 anos no Estado de São Paulo ${ }^{17}$.

As alterações no padrão respiratório e/ou ventilatório podem influenciar a coordenação entre deglutição e respiração, sendo que a sincronia entre

\section{A avaliação fonoaudiológica na UTI é indicada especialmente para pacientes \\ com suspeita de disfagia...}

elas é essencial para a proteção adequada de via aérea inferior. Portanto, pacientes com dificuldades respiratórias representam um grupo de risco expressivo para disfagia ${ }^{20}$.

0 fato das chances estatísticas de pacientes portadores de IC estarem no grupo de risco para disfagia se deve à dificuldade respiratória ser um dos sintomas mais comuns da IC, sendo esta a causadora de exacerbação respiratória e, por esse motivo, ela altera/descoordena os mecanismos de deglutição do paciente, principalmente no momento da pausa apneica ${ }^{15}$.

Houve maior número de pareceres para pacientes internados em UTIs. A avaliação fonoaudiológica na UTI é indicada especialmente para pacientes com suspeita de disfagia, considerando o ACE, a IOT prolongada e a TQT os principais fatores de risco, visando a identificar as possíveis alterações funcionais que interferem nas fases oral e faríngea da deglutição ${ }^{21}$.

Em estudos já realizados 22 se observa que houve aumento do número de fonoaudiólogos nas unidades de urgência e emergência no período analisado, sendo que, em 2005, dos 9.610 fonoaudiólogos atuantes nos estados brasileiros, 38 estavam alocados em unidades de urgência e emergência; em 2011, eles passaram para 69 , concentrados principalmente na região Sudeste, porém, uma quantidade insuficiente para atender à demanda existente no país.

Dos pacientes que passaram por algum procedimento cirúrgico, $57 \%$ foram avaliados pelo fonoaudiólogo. 0 tempo médio de espera para 0 atendimento dessa especialidade foi de $1 \mathrm{dia}$, sendo que $71 \%$ dos pacientes foram avaliados em menos de 24 horas após a solicitação.

Quanto à origem dos encaminhamentos, os médicos são os que mais realizam. 0 índice de encaminhamentos médicos talvez se justifique pelo fato de serem os profissionais que sabem o melhor momento do paciente para uma possível solicitação 
de avaliação especializada ${ }^{23}$.

A dificuldade de deglutir (96\%) foi a principal queixa fonoaudiológica relatada pelos profissionais quando solicitado parecer, em seguida aparecem as alterações vocais, especificamente a rouquidão, com apenas $4 \%$. 0 s resultados verificados neste estudo evidenciaram que a amostra foi constituída por idosos, população na qual o efeito do envelhecimento no processo de deglutição, associado a alterações da saúde, tornam o mecanismo mais vulnerável a distúrbios como a disfagia ${ }^{24}$.

Pacientes submetidos a cirurgias consideradas de grande porte podem apresentar inúmeras complicações, entre elas as de causa respiratória, que culminam com a necessidade de cuidados intensivos, bem como suporte ventilatório por tempo prolongado, isso pode acarretar alterações vocais ${ }^{25}$.

Entretanto, um dos sintomas mais frequentes apresentados pelos pacientes no periodo pósoperatório é a rouquidão, que pode estar presente em $14,4 \%$ a $50 \%$ dos pacientes submetidos a intubação orotraqueal. Esse sintoma, na maioria das vezes, é temporário, durando de 2 a 3 dias $^{26}$.

Em alguns estudos ${ }^{24}$ se observou que as vias de alimentação mais prevalentes foram sonda nasogástrica (SNG) $(82,53 \%)$ e a SNE $(11,79 \%)$. 0 que se contrapõe aos dados obtidos neste estudo, onde prevaleceu o maior número de pacientes usando SNE $(86 \%)$. Possivelmente por serem as vias mais comuns em quadros de distúrbios da deglutição crônicos e/ ou irreversíveis da amostra estudada.

0 s autores concluíram em suas pesquisas que 0 uso de VAA em pacientes sugere que parte dessa população seja disfágica ou apresente riscos para 0 desenvolvimento desse distúrbio ${ }^{24}$.

0 tempo médio de utilização da VAA da população em estudo foi de 23,8 dias. Estudos mostram ${ }^{27}$ que a média do tempo de uso de VAA, até a data do primeiro exame, foi de 16,4 dias.

Desse modo, pesquisadores ${ }^{28}$ relatam que 0 maior tempo de internação, aparentemente, tem uma relação direta com a necessidade de VAA, ou seja, quanto maior o tempo de internação, maior é a necessidade de VAA. Além disso, a patologia de base também influencia isso.

Neste estudo, houve prevalência no número de pacientes que não foram liberados da alimentação por via oral. A frequência foi semelhante à identificada em outro estudo ${ }^{29}$, onde foram avaliados todos os pacientes que deram entrada em hospital universitário de referência no período de um ano,

\section{...um dos sintomas \\ mais frequentes \\ apresentados pelos \\ pacientes no período \\ pósoperatório é a rouquidão...}

com análise objetiva da deglutição (61 pacientes). Foi observada incidência de disfagia em $76,5 \%$ dos pacientes avaliados.

Em estudo retrospectivo, realizado em um hospital geral, registrou-se a incidência de disfagia em 100 pacientes admitidos em período de 6 meses e determinou-se a presença de disfagia em $45 \%$ dos pacientes. Em outro estudo, que analisou 128 pacientes, no período de um ano, encontrou-se disfagia em $51 \%$ dos pacientes ${ }^{30}$.

Quanto ao uso de oxigenoterapia, encontrou-se uso de VM em $25 \%$ dos pacientes. Estudo mostra que $50 \%$ dos pacientes submetidos a VM por período superior a 7 dias desenvolvem anormalidades eletrofisiológicas, com incidência média de $25 \%$ de fraqueza muscular e mais de $20 \%$ de incidência de disfagia ${ }^{27}$.

Em relação aos aspectos fonoaudiológicos mais observados durante avaliação, destacam-se tosse, engasgos e escape precoce do alimento, seguido por alteração de tônus, força, mobilidade e sensibilidade oral diminuída e saída de saliva corada ou alimento corado em $18 \%$ dos pacientes avaliados.

No entanto, pacientes submetidos a intubação orotraqueal apresentam alterações frequentes, como penetração e aspiração laríngea, sendo comum a presença de mais de uma alteração para cada paciente $\mathrm{e}^{27}$.

Estudos recentes relatam que, dentre os efeitos que a intubação prolongada pode acarretar, as alterações sensoriais e motoras são justificadas por perda prematura do bolo e penetração e/ou aspiração antes, durante e/ou após a deglutição ${ }^{20}$.

Desse modo, a penetração e a aspiração laríngeas ocorrem pela diminuição ou falta de coordenação do fechamento laríngeo durante o processo da deglutição; são fatores de risco para pneumonias, podendo levar o paciente a comprometimentos pulmonares letais ${ }^{27}$.

Em relação à avaliação fonoaudiológica realizada 
em 17 pacientes, internados e submetidos a VM, pôde-se observar que os parâmetros referentes às estruturas orofaríngeas alteradas foram tonicidade de língua $(82,4 \%)$ e de lábios $(76,5 \%)$, amplitude reduzida do movimento de língua $(70,6 \%)$, de lábios $(64,7 \%)$ e de mandíbula $(58,8 \%)$.

0 s resultados demonstraram que os pacientes traqueostomizados dependentes do ventilador apresentam comprometimento das estruturas orofaríngeas como atrofia muscular e diminuição da mobilidade, possivelmente interferindo na coordenação da dinâmica da deglutição.

\section{CONCLUSÃO}

Embora o número de fonoaudiólogos seja insuficiente para cobrir a demanda de que o serviço necessita, atualmente, com apenas 3 fonoaudiólogas, o tempo de espera para cumprimento dos pareceres se mostrou curto, ou seja, os pacientes não esperavam receber o atendimento fonoaudiológico em poucos dias.

0 impacto da fonoaudiologia no ambiente hospitalar vem sendo comprovado cientificamente, proporcionando, inclusive, redução de custos hospitalares, visto que muitos dos pacientes conseguem progredir sua dieta para uma via oral segura, sendo ainda minimizados os riscos de complicações clínicas por broncoaspirações.

Neste estudo, as informações obtidas reforçam a importância do fonoaudiólogo junto à equipe multiprofissional, otimizando a proteção das vias aéreas e introduzindo de modo seguro a dieta por via oral, refletindo acerca da necessidade de divulgação e ampliação do serviço de fonoaudiologia dentro do hospital.

Mostra-se necessário ampliar este estudo para que a amostra tenha significância estatística e para que sejam cruzados os dados do estudo.

\section{CONTRIBUIÇÃO DOS AUTORES}

\section{Bruna Rafaelle Pereira Ibiapina Coelho,} Luana Pereira Ibiapina Coelho, Elizabeth Zayra Torres Sousa e Ana Virginia Sales Monte Costa contribuíram com a realização da pesquisa, além do delineamento do estudo e a revisão crítica do manuscrito. Antonio Tiago da Silva Souza e Camila Fernandes Mendes contribuíram com o delineamento do estudo e a redação do manuscrito.

\section{REFERÊNCIAS}

1. Castro LCV, Franceschini SCC, Priore SE, Peluzio MCG. Nutrição e doenças cardiovasculares: os marcadores de risco em adultos. Rev Nutr [serial on the internet]. 2004 [cited 2015 May 5];17(3):36977. Available from: http://www.scielo.br/pdf/rn/ v17n3/21886.pdf

2. Batista SRR, Jardim PCBV, Sousa ALL, Salgado CM. Hospitalizações por condições cardiovasculares sensíveis à atenção primária em municípios goianos. Rev Saúde Pública [serial on the internet]. 2012 [cited 2015 May 5];46(1):34-42. Available from: http://www.scielo.br/pdf/rsp/v46n1/2872.pdf

3. Werle RW, Steidl EMS, Mancopes R. Fatores relacionados à disfagia orofaríngea no pósoperatório de cirurgia cardíaca: revisão sistemática. CoDAS [serial on the internet]. 2016 [cited 2016 Jan 27];28(5):646-52. Available from: http:// www.scielo.br/pdf/codas/v28n5/2317-1782codas-2317-178220162015199.pdf

4. Mesquita RB, Morano MTAP, Landim FLP, Collares PMC, Pinto JMS. Rede de apoio social e saúde de idosos pneumopatas crônicos. Ciênc Saúde Colet [serial on the internet]. 2012 [cited 2015 May 5];17(5):112533. Available from: http://www.scielo.br/pdf/csc/ v17n5/a06v17n5.pdf

5. Sociedade Brasileira de Pneumologia e Tisiologia. II Consenso Brasileiro sobre Doença Pulmonar Obstrutiva Crônica (DPOC). Caracterização da doença pulmonar obstrutiva crônica (DPOC): definição, epidemiologia, diagnóstico e estadiamento. J Bras Pneumol. 2004;30(5):10-4. Available from: http://www.jornaldepneumologia.com.br/pdf/ suple $124 \quad 40$ dpoc completo finalimpresso.pdf

6. Volpe RCC, Fitipaldi R, Silva RWP, Lehn CN, Fava AS. Associação de doença pulmonar obstrutiva crônica (DPOC) e complicações em cirurgia de cabeça e pescoço. Braz J 0torhinolaryngol [serial on the internet]. 2011 [cited 2015 May 6];77(1):125-8. Available from: http://www.scielo.br/pdf/bjorl/ v77n1/v77n1a21.pdf

7. Silva DR, Gazzana MB, Knorst MM. Valor dos achados clínicos e da avaliação funcional pulmonar pré-operatórios como preditores das complicações pulmonares pós-operatórias. Rev Assoc Méd Bras [serial on the internet]. 2010 [cited 2015 May 6];56(5):551-7. Available from: http://www.scielo. $\mathrm{br} / \mathrm{pdf} / \mathrm{ramb} / \mathrm{v} 56 \mathrm{n} 5 / \mathrm{v} 56 \mathrm{n} 5 \mathrm{a} 16 . \mathrm{pdf}$

8. Chaves RD, Carvalho CRF, Cukier A, Stelmach R, Andrade CRF. Sintomas indicativos de disfagia em portadores de DPOC. J Bras Pneumol [serial on the internet]. 2011 [cited 2015 May 10];37(2):176-83. Available from: http://www.scielo.br/pdf/jbpneu/ v37n2/v37n2a07.pdf 
9. O'Kane L, Groher M. Oropharyngeal dysphagia in patients with chronic obstructive pulmonary disease: a systematic review. Rev CEFAC [serial on the internet]. 2009 [cited 2016 Jan 27];11(3):449506. Available from: http://www.scielo.br/pdf/ rcefac/v11n3/42-09.pdf

10. Leite ICG, Simões AG, Clemente MCKC, Martins LS, Bittar AS, Bittar CL, et al. Fonoaudiologia hospitalar. Jornal Brasileiro de Fonoaudiologia [serial on the internet]. 2003 [cited 2015 May 10];4(17):[about 6 screens]. Available from: https://www.dtscience. com/wp-content/uploads/2015/10/FonoaudiologiaHospitalar.pdf

11. Monti MMF, Botega MBS, Lima MCMP, Kubota SMP. Demanda para intervenção fonoaudiológica em uma unidade neonatal de um hospital-escola. Rev CEFAC [serial on the internet]. 2013 [cited 2015 May 10];15(6):1540-51. Available from: http://www. scielo.br/pdf/rcefac/v15n6/v15n6a17.pdf

12. Medeiros EA, Maia RM, Cedro MO, Barbosa MLC, Correia RBF, Tavares PMB, et al. A inserção da fonoaudiologia na Estratégia Saúde da Família: vivências em Sobral-CE. Sanare (Sobral, Online) [serial on the internet]. 2009 [cited 2016 May 3];8(2):7-15. Available from: https://sanare. emnuvens.com.br/sanare/article/viewFile/14/11

13. Silverio CC, Hernandez AM, Goncalves MIR. Ingesta oral do paciente hospitalizado com disfagia orofaríngea neurogênica. Rev CEFAC [serial on the internet]. 2010 [cited 2015 May 15];12(6):964-70. Available from: http://www.scielo.br/pdf/rcefac/ v12n6/81-09.pdf

14. Faria CF, Pessoa ACN, Araujo LI, Paiva MLF. Perfil do paciente atendido pela fonoaudiologia na unidade de urgência e emergência de um hospital universitário. Audiol Commun Res [serial on the internet]. 2013 [cited 2015 May 10];18(4):30813. Available from: http://www.scielo.br/pdf/acr/ v18n4/12.pdf

15. Bassi D, Furkim AM, Silva CA, Coelho MSPH, Rolim MRP, Alencar MLA, et al. Identificação de grupos de risco para disfagia orofaríngea em pacientes internados em um hospital universitário. Rev Soc Bras Fonoaudiol [serial on the internet]. 2014 [cited 2015 Jun 10];26(1):17-27. Available from: http:// www.scielo.br/pdf/codas/v26n1/pt 2317-1782codas-26-01-00017.pdf

16. Rosado CV, Amaral LKM, Galvão AP, Guerra SD, Furia CLB. Avaliação da disfagia em pacientes pediátricos com traumatismo crânio-encefálico. Rev CEFAC [serial on the internet]. 2005 [cited 2015 Jun 12];7(1):34-41. Available from: http://www.redalyc. org/pdf/1693/169320490004.pdf
17. Francisco PMSB, Donalisio MR, Lattorre MRDO. Internações por doenças respiratórias em idosos e a intervenção vacinal contra influenza no Estado de São Paulo. Rev Bras Epidemiol [serial on the internet]. 2004 [cited 2016 Mar 2];7(2):220-7. Available from: http://www.scielo.br/pdf/rbepid/v7n2/18.pdf

18. Costa RG, Souza LBR. Perfil dos usuários e da demanda pelo serviço da clínica-escola de fonoaudiologia da UFBA. Rev Ciênc Méd Biol [serial on the internet]. 2009 [cited 2016 Mar 2];8(1):539. Available from: https://portalseer.ufba.br/index. $\mathrm{php/cmbio/article/view/4376/3200}$

19. Assis MMA, Jesus WLA. Acesso aos serviços de saúde: abordagens, conceitos, políticas e modelo de análise. Ciênc Saúde Colet [serial on the internet]. 2012 [cited 2016 Mar 2];17(11):2865-75. Available from: http://www.scielo.br/pdf/csc/v17n11/ v17n11a02.pdf

20. Furmann N, Costa FM. Critérios clínicos utilizados por profissionais para liberação de dieta via oral em pacientes adultos hospitalizados. Rev CEFAC [serial on the internet]. 2015 [cited 2016 Mar 4];17(4):127887. Available from: http://www.scielo.br/pdf/ rcefac/v17n4/1982-0216-rcefac-17-04-01278.pdf

21. Padovani AR, Andrade CRF. Perfil funcional da deglutição em unidade de terapia intensiva clínica. Einstein (São Paulo) [serial on the internet]. 2007 [cited 2016 Mar 4];5(4):358-62. Available from: http://www.fonovim.com.br/arquivos/784a647f7e 75 9641c32d76551d8be259-Perfil-funcional-da-degluti---o-em-unidade-de.pdf

22. Costa KN, Guimarães VC. Fonoaudiologia nos serviços de urgência e emergência do Brasil: série histórica de 2005 a 2011. Distúrb Comun [serial on the internet]. 2012 [cited 2016 Mar 4];24(1):69-75. Available from: http://revistas.pucsp.br/index.php/ dic/article/view/9707/7211

23. Hage SRV, Faiad LNV. Perfil de pacientes com alteração de linguagem atendidos na clínica de diagnóstico dos distúrbios da comunicação. Rev CEFAC [serial on the internet]. 2005 [cited 2016 Mar 4];7(4):433-40. Available from: http://www.redalyc. org/pdf/1693/169320507006.pdf

24. Nogueira SCJ, Carvalho APC, Melo CB, Morais EPG, Chiari BM, Gonçalves MIR. Perfil de pacientes em uso de via alternativa de alimentação internados em um hospital geral. Rev CEFAC [serial on the internet]. 2013 [cited 2016 Mar 7];15(1):94-104. Available from: http://www.scielo.br/pdf/rcefac/v15n1/13311.pdf

25. Arcêncio L, Souza MD, Bortolin BS, Fernandes CM, Rodrigues AJ, Evora PRB. Cuidados pré e pós-operatórios em cirurgia cardiotorácica: uma abordagem fisioterapêtica. Rev Bras Cir Cardiovasc [serial on the internet]. 2008 [cited 2016 Mar 7];23(3):400-10. Available from: http://www. scielo.br/pdf/rbccv/v23n3/v23n3a19.pdf 
26. Mota LAA, Carvalho GBC, Brito VA. Complicações laríngeas por intubação orotraqueal: revisão da literatura. Int Arch 0torhinolaryngol [serial on the internet]. 2012 [cited 2016 Mar 7];16(2):23645. Available from: http://www.scielo.br/pdf/iao/ v16n2/v16n2a14.pdf

27. Kunigk MRG, Chehter E. Disfagia orofaríngea em pacientes submetidos à entubação orotraqueal. Rev Soc Bras Fonoaudiol [serial on the internet]. 2007 [cited 2016 Mar 10];12(4):287-91. Available from: http://www.scielo.br/pdf/rsbf/v12n4/v12n4a06

28. Gregório JGR, Valério KD, Andrade WTL. Uso de via alimentar alternativa em pacientes admitidos na unidade de terapia intensiva de um hospital público da cidade de João Pessoa/PB [document on the internet]. 2010 [cited 2019 May 20]. Available from: http://www.redentor.inf.br/arquivos/pos/ publicacoes/15082012TCC\%20Jose $\% 20$ Geraldo.pdf

29. Schelp A0, Cola PC, Gatto AR, Silva RG, Carvalho LR. Incidência de disfagia orofaríngea após acidente vascular encefálico em hospital público de referência. Arq Neuropsiquiatr [serial on the internet]. 2004 [cited 2016 Mar 10];62(2):503-6. Available from: http://www.scielo.br/pdf/anp/v62n2b/a23v622b.pdf

30. Maciel JRV, Oliveira CJR, Tada CMP. Associação entre risco de disfagia e risco nutricional em idosos internados em hospital universitário de Brasília. Rev Nutr [serial on the internet]. 2008 [cited 2016 Mar 10];21(4):411-21. Available from: http://www. scielo.br/pdf/rn/v21n4/v21n4a05.pdf
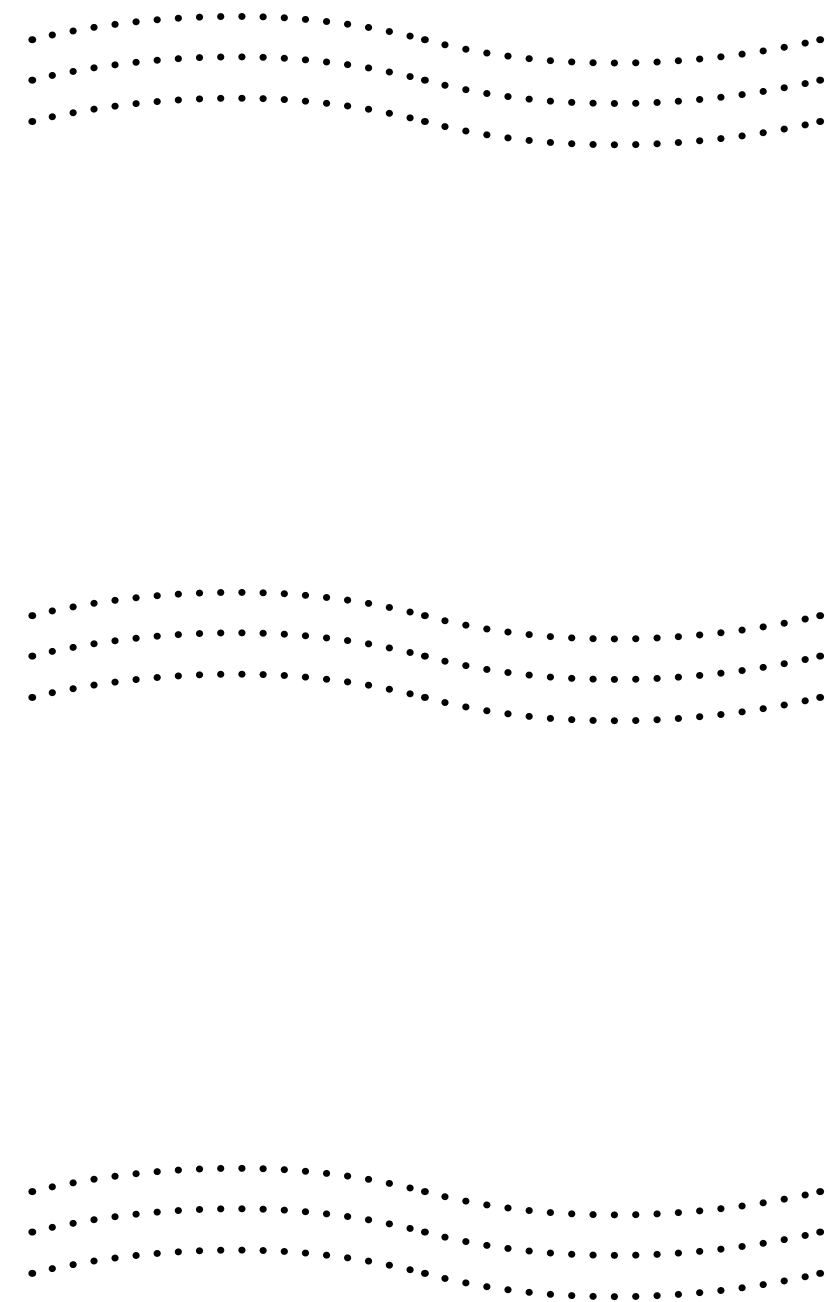
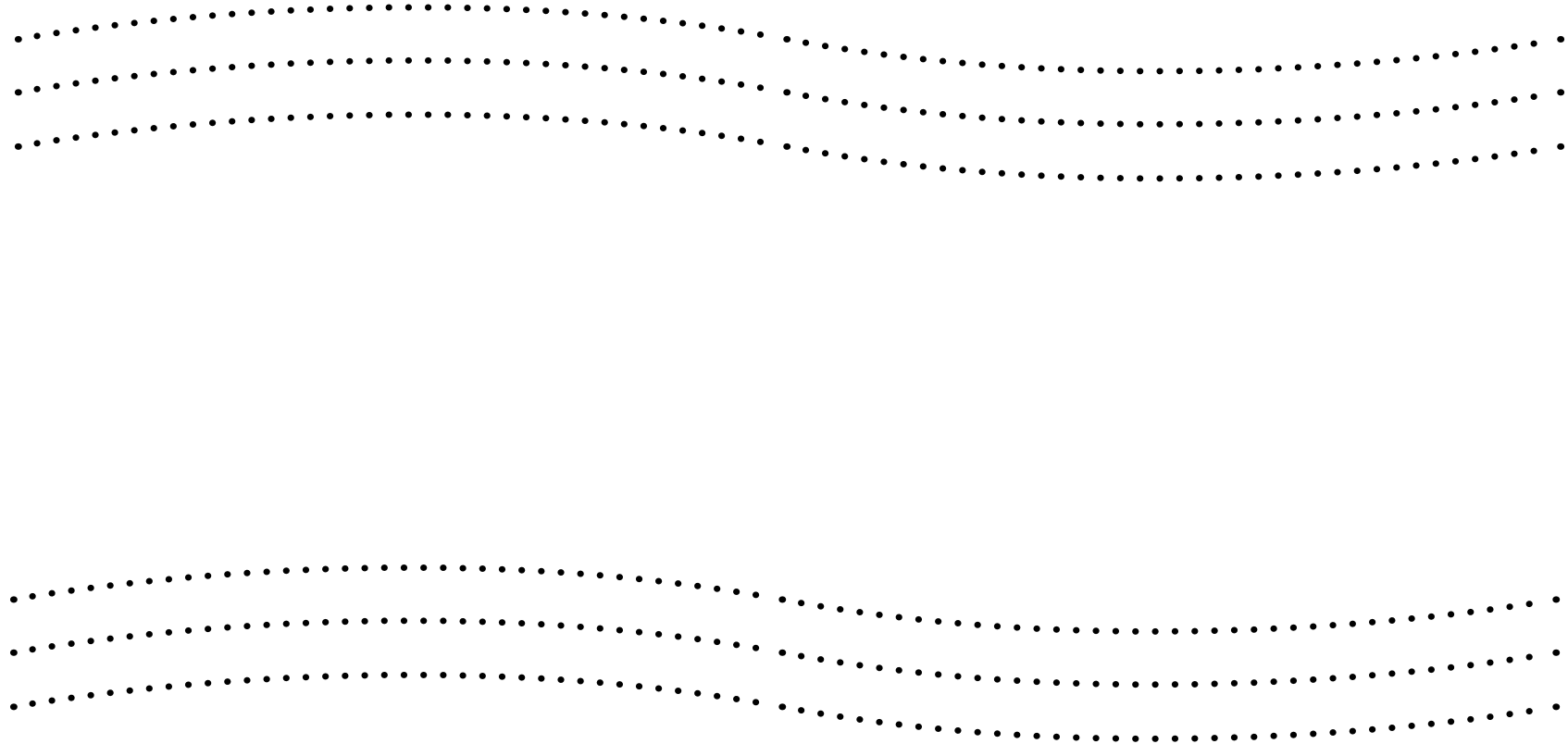